\title{
AVALIAÇÃO DO ESPECTRO DE GOTAS DE PONTAS DE PULVERIZAÇÃO HIDRÁULICAS UTILIZANDO A TÉCNICA DA DIFRAÇÃO DO RAIO LASER
}

\section{JOÃO P. A. R. DA CUNHA ${ }^{1}$, MAURI M. TEIXEIRA ${ }^{2}$, HAROLDO C. FERNANDES ${ }^{3}$}

RESUMO: As pontas de pulverização são os componentes mais importantes dos pulverizadores na aplicação de agrotóxicos. Dessa forma, este trabalho teve como objetivo avaliar o espectro de gotas de pontas de pulverização hidráulicas de jato plano (standard e antideriva) e de jato cônico vazio, disponíveis no mercado, submetidas a diferentes pressões da calda. Em ambiente controlado, avaliou-se o espectro de gotas, por meio de um analisador a laser de gotas em tempo real, nas pressões de 200; 300 e $400 \mathrm{kPa}$ para as pontas de jato plano, e de 400; 500 e $600 \mathrm{kPa}$ para as pontas de jato cônico. Os resultados permitiram concluir que: o espectro de gotas foi influenciado pela vazão nominal das pontas e pela pressão da calda; o potencial de deriva das pontas de jato plano standard e de jato cônico vazio foi alto; o uso de pontas de pulverização antideriva dotadas de préorifício aumentou o diâmetro das gotas, constituindo-se, portanto, em fator auxiliar para a redução da deriva nas pulverizações, e a avaliação do espectro de gotas empregando-se a técnica da difração do raio laser mostrou-se rápida e precisa.

PALAVRAS-CHAVE: tecnologia de aplicação, agrotóxicos, pulverizadores.

\section{DROPLET SPECTRUM EVALUATION OF HYDRAULIC SPRAY NOZZLES USING LASER DIFFRACTION TECHNIQUE}

\begin{abstract}
The spray nozzles are one of the most important components of pesticide hydraulic sprayer. This study evaluated the droplet spectrum of hydraulic flat fan (standard and low drift) and hollow cone spray nozzles, available in the market, under different working pressures. In laboratory, droplet spectrum, using a real time laser particle size analyzer, was measured with the operating pressure of 200; 300 and $400 \mathrm{kPa}$, for flat fan, and 400; 500 and $600 \mathrm{kPa}$, for hollow cone. The results showed that: the droplet spectrum was affected by the nominal flow and by the liquid pressure; the drift potential of standard flat fan and hollow cone spray nozzles was high; the use of low drift nozzles increased the droplet diameter, which was an effective factor in drift reduction, and the technique of laser diffraction provides a robust means of measuring the droplet size produced by spray systems.
\end{abstract}

KEYWORDS: spray technology, pesticides, sprayers.

\section{INTRODUÇÃO}

O objetivo da tecnologia de aplicação de agrotóxicos é colocar a quantidade certa de ingrediente ativo no alvo, com a máxima eficiência e de maneira econômica, afetando o mínimo possível o ambiente (MATTHEWS, 2002). Muitas vezes, entretanto, parte do produto aplicado não atinge o alvo, principalmente em razão da deriva que, além dos danos que pode causar em culturas adjacentes, também pode contaminar a água, o solo e os alimentos.

Entre as diferentes técnicas de aplicação de agrotóxicos, as que se baseiam na pulverização hidráulica são as mais difundidas, graças à flexibilidade que oferecem em diferentes situações (TEIXEIRA, 1997). Existem vários tipos de pulverizadores hidráulicos, que vão desde os mais

\footnotetext{
${ }^{1}$ Eng ${ }^{0}$ Agrícola, Prof. Adjunto, Instituto de Ciências Agrárias, Universidade Federal de Uberlândia, Câmpus Umuarama, Uberlândia - MG, Fone: (0XX34) 3218.2225, jpcunha@iciag.ufu.br

${ }^{2}$ Eng ${ }^{\mathrm{o}}$ Agrônomo, Prof. Adjunto, Departamento de Engenharia Agrícola, UFV, Viçosa - MG.

${ }^{3}$ Eng $^{\circ}$ Agrícola, Prof. Adjunto, Departamento de Engenharia Agrícola, UFV, Viçosa - MG.

Recebido pelo Conselho Editorial em: 21-6-2005
}

Aprovado pelo Conselho Editorial em: 21-3-2006 
simples, do tipo costal, utilizado em pequenas áreas, até os equipamentos mais sofisticados, como os pulverizadores de barra autopropelidos. Nesses equipamentos, os bicos de pulverização representam um dos principais componentes, pois influenciam diretamente na qualidade e na segurança da aplicação. Genericamente, denomina-se bico ao conjunto de peças colocado no final do circuito hidráulico, por meio do qual a calda é fragmentada em gotas (CHRISTOFOLETTI, 1999). O bico consiste de várias partes, sendo a ponta de pulverização a mais importante.

A correta aplicação de agrotóxicos utilizando pulverizadores de barra tratorizados somente é possível quando se dispõe de pontas de pulverização que propiciem distribuição satisfatória e espectro de gotas uniforme e de tamanho adequado. Uma aplicação eficiente requer cobertura adequada da superfície-alvo com gotas de tamanho apropriado. No caso de serem produzidas gotas muito grossas, não ocorre boa cobertura da superfície, tampouco boa uniformidade de distribuição e deposição. Essas gotas, devido ao peso, normalmente não se aderem à superfície da folha e terminam no solo (LEFEBVRE, 1989).

No caso de gotas muito finas, geralmente, se consegue boa cobertura superficial e uniformidade de distribuição da calda, mas essas gotas podem evaporar em condições de baixa umidade relativa ou serem levadas pela corrente de ar. Vários pesquisadores consideram que gotas menores que $100 \mu \mathrm{m}$ são facilmente carregadas pelo vento, sofrendo mais intensamente a ação dos fenômenos climáticos (SUMNER, 1997; MURPHY et al., 2000, e WOLF, 2000).

Assim, durante as aplicações de agrotóxicos, deve-se cuidar para que não sejam produzidas gotas muito grossas, nem muito finas. É preciso conhecer as características técnicas das pontas visando a sua correta seleção e, com isso, aplicações eficientes e seguras ambientalmente. Nas pontas tradicionais, que operam com pressão hidráulica, a formação de gotas é bastante desuniforme, dificultando, muitas vezes, boa cobertura do alvo.

Dessa forma, este trabalho teve como objetivo avaliar o espectro de gotas de pontas de pulverização hidráulicas de jato plano e de jato cônico vazio, disponíveis no mercado, submetidas a diferentes pressões da calda.

\section{MATERIAL E MÉTODOS}

Os ensaios foram realizados no Laboratório de Controle Ambiental do Departamento de Engenharia Química da Universidade Federal de São Carlos. Foram avaliadas cinco pontas de pulverização hidráulicas, três de jato plano e duas de jato cônico vazio, fabricadas em alumina revestida por poliacetal: API 110-02, API 110-04, ADI 110-02, ATR Brown e ATR Red (todas fabricadas pela Albuz, Ceramiques Techniques Desmarquest, Evreux, France). As pontas de jato plano standard API (alumina plástico ISO) e de jato plano antideriva ADI (Alumina Drift ISO) são de uso geral e, de acordo com o fabricante, proporcionam aplicações uniformes quando os jatos de pulverização se sobrepõem. As pontas de jato cônico vazio ATR (alumina turbulência) são indicadas, de acordo com o fabricante, para aplicações de fungicidas e inseticidas com pressão superior a $300 \mathrm{kPa}$, em barras de pulverização.

Para a avaliação do espectro de gotas, empregaram-se as pressões de 200; 300 e $400 \mathrm{kPa}$ para as pontas de jato plano, e de 400; 500 e $600 \mathrm{kPa}$ para as de jato cônico vazio. Os seguintes parâmetros foram tomados: $\mathrm{D}_{\mathrm{v} 0,1}$ (diâmetro de gota tal que $10 \%$ do volume do líquido pulverizado é constituído de gotas menores que esse valor); $\mathrm{D}_{\mathrm{v} 0,5}$ (diâmetro de gota tal que $50 \%$ do volume do líquido pulverizado é constituído de gotas menores que esse valor, também conhecido como diâmetro mediano volumétrico - DMV); $\mathrm{D}_{\mathrm{v} 0,9}$ (diâmetro de gota tal que $90 \%$ do volume do líquido pulverizado é constituído de gotas menores que esse valor); SPAN (amplitude relativa) e percentagem do volume de gotas com diâmetro inferior a 100; 150 e $200 \mu \mathrm{m}$.

A amplitude relativa (SPAN) foi determinada utilizando-se da seguinte equação:

$$
\mathrm{SPAN}=\frac{\mathrm{D}_{\mathrm{v} 0,9}-\mathrm{D}_{\mathrm{v} 0,1}}{\mathrm{D}_{\mathrm{v} 0,5}}
$$


O espectro da população de gotas foi determinado de forma direta, utilizando-se de analisador de gotas em tempo real. Esse analisador baseia-se na medição da luz (feixe de raio laser) difratada durante a passagem das gotas pulverizadas pela região de amostragem do aparelho (SCHICK, 1997). O equipamento dispõe de unidade óptica que detecta o padrão de difração da luz ao passar por um conjunto de partículas, apresentando capacidade de mensuração de gotas com diâmetro entre 0,5 e $1.000 \mu \mathrm{m}$. O desvio que o feixe de laser sofre, depende do tamanho da partícula. Quanto menor a partícula, maior é o grau de difração que o raio de luz sofre.

Empregou-se o equipamento Spraytec (Malvern Spraytec Real Time Droplet Sizing System, Malvern Instruments, Malvern, England), com diâmetro do feixe do laser de $10 \mathrm{~mm}$, lente focal de $200 \mathrm{~mm}$, comprimento de onda do laser de $670 \mathrm{~nm}$ e ponte óptica de base longa. Inicialmente, verificou-se o alinhamento do feixe óptico para garantir o seu correto posicionamento no sistema detector; monitorou-se o sistema de fundo (segundo plano), medida da luz desviada no sistema óptico e da contaminação das janelas por partículas, e procedeu-se à calibração do aparelho, utilizando-se do retículo-padrão RS-3 (retículo aprovado pelo ASTM - American Society for Testing and Materials - para calibração de instrumentos de análise de partículas baseados na técnica da difração do laser). De acordo com o fabricante, o instrumento apresenta $3 \%$ de acurácia e $1 \%$ de precisão, quando se utiliza o retículo-padrão. Para a análise dos dados, fez-se uso do programa computacional RTSizer ${ }^{\mathrm{TM}}$ (Insitec, Malvern, England).

Durante a determinação do espectro de gotas, utilizou-se de equipamento de pulverização costal, a pressão constante $\left(\mathrm{CO}_{2}\right)$, montado de tal forma que todo o jato pulverizado passasse transversalmente por meio do feixe luminoso do analisador. Isso permitiu a obtenção direta do espectro de gotas para cada condição desejada. $\mathrm{O}$ bico de pulverização ficou posicionado $30 \mathrm{~cm}$ acima do feixe óptico, e o líquido de teste foi água pura fornecida pela empresa local de abastecimento, conforme metodologia apresentada pela ASAE (2000).

Todos os ensaios foram realizados em ambiente protegido, e as condições ambientais foram: temperatura do ar inferior a $28{ }^{\circ} \mathrm{C}$, umidade relativa do ar superior a $60 \%$ e ausência de ventos. Os manômetros utilizados, com capacidade nominal de $2.059,4 \mathrm{kPa}\left(21 \mathrm{kgf} \mathrm{cm}^{-2}\right)$ e resolução de 98,1 $\mathrm{kPa}\left(1 \mathrm{kgf} \mathrm{cm}{ }^{-2}\right)$, foram previamente calibrados por meio de estrutura de reação dotada de massaspadrão. Para a análise estatística dos dados de espectro de gotas de cada ponta, utilizou-se do delineamento inteiramente casualizado, com cinco repetições. Utilizou-se do teste de Tukey, a 5\% de probabilidade, para a comparação das médias dos tratamentos. As análises estatísticas foram realizadas empregando-se o programa estatístico SAEG 8.0.

\section{RESULTADOS E DISCUSSÃO}

Encontram-se, na Tabela 1, os diâmetros médios dos volumes acumulados de 10\%, 50\% e $90 \%$, a amplitude relativa e a vazão das pontas avaliadas nas diferentes pressões. Em geral, médias de DMV inferiores a $250 \mu \mathrm{m}$ indicam risco potencial de deriva; no entanto, médias de DMV superiores a $500 \mu \mathrm{m}$ sugerem problemas de escorrimento (TEIXEIRA, 1997).

As pontas apresentaram espectros de gotas diferenciados. Nas pontas de jato plano, o diâmetro das gotas diminuiu com o incremento da pressão e aumentou, nas pontas standard, com o crescimento da vazão nominal. Com exceção do diâmetro mediano volumétrico da ponta ATR Brown, o aumento da pressão nas pontas de jato cônico vazio não influenciou no diâmetro das gotas, mas este teve aumento com o crescimento da vazão nominal. O aumento do orifício de saída das pontas proporcionou menor quebra do líquido, o que gera gotas de tamanho maior.

Pequenas variações no tamanho das gotas provenientes de pontas de jato cônico vazio com a variação da pressão do líquido também foram constatadas por RAETANO (1996). Essas pontas apresentam faixa operacional de pressão maior. Portanto, para obter variações do tamanho das gotas, deve-se aumentar muito a pressão, o que nem sempre é desejável em virtude do desgaste do equipamento. 
TABELA 1. Distribuição volumétrica por classe de tamanho e amplitude relativa de gotas pulverizadas com pontas de jato plano standard, jato plano antideriva e jato cônico vazio, em diferentes pressões ${ }^{1}$.

\begin{tabular}{lcccccc}
\hline \multicolumn{1}{c}{ Ponta } & $\begin{array}{c}\text { Pressão } \\
(\mathrm{kPa})\end{array}$ & $\begin{array}{c}\text { Vazão } \\
\left(\mathrm{L} \mathrm{min}^{-1}\right)\end{array}$ & $\begin{array}{c}\mathrm{D}_{\mathrm{v} 0,1} \\
(\mu \mathrm{m})\end{array}$ & $\begin{array}{c}\mathrm{D}_{\mathrm{v} 0,5} \\
(\mu \mathrm{m})\end{array}$ & $\begin{array}{c}\mathrm{D}_{\mathrm{v} 0,9} \\
(\mu \mathrm{m})\end{array}$ & SPAN \\
\hline Jato Plano Standard & 200 & 0,63 & $81 \mathrm{a}$ & $164 \mathrm{a}$ & $330 \mathrm{a}$ & 1,52 \\
API 110-02 & 300 & 0,80 & $64 \mathrm{~b}$ & $134 \mathrm{~b}$ & $280 \mathrm{ab}$ & 1,61 \\
\hline \multirow{2}{*}{ Jato Plano Standard } & 400 & 0,91 & $57 \mathrm{c}$ & $124 \mathrm{~b}$ & $256 \mathrm{~b}$ & 1,60 \\
API 110-04 & 200 & 1,24 & $91 \mathrm{a}$ & $214 \mathrm{a}$ & $400 \mathrm{a}$ & $1,45 \mathrm{c}$ \\
\hline \multirow{2}{*}{ Jato Plano Antideriva } & 400 & 1,53 & $75 \mathrm{~b}$ & $180 \mathrm{~b}$ & $378 \mathrm{~b}$ & $1,68 \mathrm{~b}$ \\
ADI 110-02 & 200 & 1,77 & $67 \mathrm{~b}$ & $163 \mathrm{c}$ & $365 \mathrm{c}$ & $1,84 \mathrm{a}$ \\
\hline \multirow{2}{*}{ Jato Cônico Vazio } & 400 & 0,62 & $113 \mathrm{a}$ & $322 \mathrm{a}$ & $434 \mathrm{a}$ & $1,00 \mathrm{~b}$ \\
ATR Brown & 400 & 0,90 & $101 \mathrm{ab}$ & $298 \mathrm{~b}$ & $429 \mathrm{a}$ & $1,10 \mathrm{~b}$ \\
& 500 & 0,43 & $49 \mathrm{~b}$ & $262 \mathrm{c}$ & $421 \mathrm{a}$ & $1,27 \mathrm{a}$ \\
\hline \multirow{2}{*}{ Jato Cônico Vazio } & 600 & 0,49 & 41 & $90 \mathrm{a}$ & 167 & 1,37 \\
ATR Red & 400 & 1,32 & 40 & $86 \mathrm{~b}$ & 160 & 1,38 \\
& 500 & 1,49 & 47 & 129 & 315 & 2,08 \\
\hline
\end{tabular}

${ }^{1} \mathrm{D}_{\mathrm{v} 0,1}$ - diâmetro de gota tal que $10 \%$ do volume do líquido pulverizado é constituído de gotas menores que esse valor; $\mathrm{D}_{\mathrm{v} 0,5}$ diâmetro de gota tal que $50 \%$ do volume do líquido pulverizado é constituído de gotas menores que esse valor (DMV); $\mathrm{D}_{\mathrm{v} 0,9}$ diâmetro de gota tal que $90 \%$ do volume do líquido pulverizado é constituído de gotas menores que esse valor, e SPAN - amplitude relativa. Para cada ponta, médias seguidas da mesma letra, nas colunas, não diferem significativamente entre si, a 5\% de probabilidade, pelo teste de Tukey. A ausência de letras indica que não houve significância no teste $\mathrm{F}$.

Na Tabela 2, é mostrada a percentagem do volume de gotas com diâmetro inferior a 100; 150 e $200 \mu \mathrm{m}$. A interpretação desses dados permite que se estime o potencial de deriva da aplicação. Quanto menor essa percentagem, menor o risco de deriva do agrotóxico durante a aplicação. Não existe valor-padrão indicativo de risco de deriva ou de aplicação segura. Em geral, valores inferiores a $15 \%$ do volume pulverizado composto por gotas com diâmetro inferior a $100 \mu \mathrm{m}$ parecem ser mais adequados a uma aplicação segura (CUNHA et al., 2003).

TABELA 2. Percentagem do volume pulverizado composto por gotas com diâmetro inferior a 100; 150 e $200 \mu \mathrm{m}$ com pontas de jato plano standard, jato plano antideriva e jato cônico vazio, em diferentes pressões.

\begin{tabular}{lcccc}
\hline \multicolumn{1}{c}{ Ponta } & $\begin{array}{c}\text { Pressão } \\
(\mathrm{kPa})\end{array}$ & $\begin{array}{c}\text { \% Volume de } \\
\text { Gotas Menores que } \\
100 \mu \mathrm{m}\end{array}$ & $\begin{array}{c}\text { \%olume de } \\
\text { Gotas Menores que } \\
150 \mu \mathrm{m}\end{array}$ & $\begin{array}{c}\% \text { Volume de } \\
\text { Gotas Menores que 200 } \mu \mathrm{m}\end{array}$ \\
\hline \multirow{2}{*}{ Jato Plano Standard } & 200 & $15,71 \mathrm{c}$ & $40,97 \mathrm{~b}$ & $60,97 \mathrm{~b}$ \\
API 110-02 & 300 & $25,57 \mathrm{~b}$ & $55,70 \mathrm{a}$ & $74,33 \mathrm{a}$ \\
& 400 & $30,72 \mathrm{a}$ & $61,68 \mathrm{a}$ & $79,04 \mathrm{a}$ \\
\hline \multirow{2}{*}{ Jato Plano Standard } & 200 & $11,32 \mathrm{c}$ & $28,67 \mathrm{c}$ & $44,64 \mathrm{c}$ \\
API 110-04 & 300 & $17,35 \mathrm{~b}$ & $38,07 \mathrm{~b}$ & $53,11 \mathrm{~b}$ \\
& 400 & $21,37 \mathrm{a}$ & $43,46 \mathrm{a}$ & $58,51 \mathrm{a}$ \\
\hline \multirow{2}{*}{ Jato Plano Antideriva } & 200 & $6,90 \mathrm{~b}$ & $16,63 \mathrm{~b}$ & $25,45 \mathrm{~b}$ \\
ADI 110-02 & 300 & $8,67 \mathrm{~b}$ & $20,57 \mathrm{~b}$ & $30,45 \mathrm{ab}$ \\
& 400 & $11,98 \mathrm{a}$ & $25,63 \mathrm{a}$ & $36,14 \mathrm{a}$ \\
\hline \multirow{2}{*}{ Jato Cônico Vazio } & 400 & $54,93 \mathrm{~b}$ & 85,05 & 93,81 \\
ATR Brown & 500 & $59,07 \mathrm{a}$ & 86,64 & 94,42 \\
& 600 & $61,20 \mathrm{a}$ & 86,83 & 94,10 \\
\hline \multirow{2}{*}{ Jato Cônico Vazio } & 400 & 35,27 & 55,80 & 69,68 \\
ATR Red & 500 & 36,66 & 57,01 & 70,74 \\
& 600 & 38,70 & 58,72 & 71,95 \\
\hline
\end{tabular}

Para cada ponta, médias seguidas da mesma letra, nas colunas, não diferem significativamente entre si, a 5\% de probabilidade, pelo teste de Tukey. A ausência de letras indica que não houve significância no teste F. 
As pontas de jato cônico vazio foram relativamente mais sujeitas à deriva que as pontas de jato plano, independentemente da pressão. Portanto, seu uso deve ser feito com critério, evitando-se situações climáticas adversas. Mesmo com a redução da pressão de operação, existe risco de perda de agrotóxico para o ambiente. Já as pontas de jato plano têm menor risco potencial de deriva, principalmente em baixas pressões. De qualquer forma, empregando-se pontas standard é preciso atentar muito para as condições de velocidade de vento, principalmente acima de $3 \mathrm{~m} \mathrm{~s}^{-1}$, pois em pressões acima de $300 \mathrm{kPa}$, mais de $15 \%$ do volume pulverizado está sujeito ao arrastamento provocado pelo vento.

As pontas antideriva contribuíram para o aumento do tamanho das gotas, diminuindo o risco potencial de deriva. O pré-orifício produz perda de carga na pressurização, com efeito inverso no diâmetro das gotas. Utilizando as pontas standard API 110-02, em média, 24\% do volume pulverizado apresentou gotas de diâmetro inferior a $100 \mu \mathrm{m}$, e com a utilização das pontas antideriva ADI 110-02, 9\%. SUMNER \& SUMNER (1999) também constataram a redução do potencial de deriva com a utilização de algumas pontas antideriva avaliadas.

\section{CONCLUSÕES}

A variação da pressão da calda influenciou mais no espectro de gotas das pontas de jato plano em relação às de jato cônico vazio.

O potencial de deriva das pontas de jato plano standard e de jato cônico vazio foi alto. O uso das pontas de pulverização antideriva dotadas de pré-orifício alterou o espectro de gotas, aumentando seu diâmetro, constituindo-se, portanto, em fator auxiliar para a redução da deriva nas pulverizações.

O incremento da vazão nominal das pontas propiciou aumento do tamanho de gotas.

A avaliação do espectro de gotas, empregando-se a técnica da difração do raio laser, mostrouse rápida e precisa.

\section{AGRADECIMENTOS}

Ao Conselho Nacional de Desenvolvimento Científico e Tecnológico (CNPq), pelo apoio concedido.

\section{REFERÊNCIAS}

ASAE S572. Spray nozzle classification by droplet spectra. In: ASAE Standards aug.99. St. Joseph, 2000. p.389-91.

CHRISTOFOLETTI, J.C. Considerações sobre tecnologia de aplicação de defensivos agrícolas. São Paulo: Teejet, 1999. 15 p.

CUNHA, J.P.A.R.; TEIXEIRA, M.M.; COURY, J.R.; FERREIRA, L.R. Avaliação de estratégias para a redução da deriva de agrotóxicos em pulverizações hidráulicas. Planta Daninha, Viçosa, v.21, n.2, p.325-32, 2003.

LEFEBVRE, A.H. Atomization and sprays. International Series: Combustion. New York: Hemisphere Publishing Corporation, 1989. 421 p.

MATTHEWS, G.A. The application of chemicals for plant disease control. In: WALLER, J.M.; LENNÉ, J.M.; WALLER, S.J. Plant pathologist's pocketbook. London: CAB, 2002. p.345-53.

MURPHY, S.D.; MILLER, P.C.H; PARKIN, C.S. The effect of boom section and nozzle configuration on the risk of spray drift. Journal of Agricultural Engineering Research, London, v.75, n.2, p.127-37, 2000. 
RAETANO, C.G. Condições operacionais de turboatomizadores na distribuição e deposição da pulverização em citros. 1996. 93 f. Tese (Doutorado em Entomologia Agrícola) - Escola Superior de Agricultura "Luiz de Queiroz”, Universidade de São Paulo, Piracicaba, 1996.

SCHICK, R.J. An engineer's practical guide drop size. Wheaton: Spraying Systems, 1997. 28 p.

SUMNER, P.E. Reducing spray drift. Georgia: University of Georgia, 1997. 11 p. (ENG97-005).

SUMNER, P.E.; SUMNER, S.A. Comparison of new drift reduction nozzles. St. Joseph: ASAE, 1999.17 p. (ASAE, Paper n.99-1156).

TEIXEIRA, M.M. Influencia del volumen de caldo y de la uniformidad de distribución transversal sobre la eficacia de la pulverización hidráulica. 1997. 310 f. Tese (Doutorado em Engenharia Rural) - Escuela Técnica Superior de Ingenieros Agrônomos, Universidad Politécnica de Madrid, Madrid, 1997.

WOLF, R.E. Strategies to reduce spray drift. Kansas: KSU, 2000. 4 p. (Application Technology Series). 